\section{A Memetic Algorithm for Multiple-Drug Cancer Chemotherapy Schedule Optimization}

Sui-Man Tse, Yong Liang, Kwong-Sak Leung, Senior Member, IEEE, Kin-Hong Lee, Senior Member, IEEE, and Tony Shu-Kam Mok

\begin{abstract}
This correspondence introduces a multidrug cancer chemotherapy model to simulate the possible response of the tumor cells under drug administration. We formulate the model as an optimal control problem. The algorithm in this correspondence optimizes the multidrug cancer chemotherapy schedule. The objective is to minimize the tumor size under a set of constraints. We combine the adaptive elitist genetic algorithm with a local search algorithm called iterative dynamic programming (IDP) to form a new memetic algorithm (MA-IDP) for solving the problem. MA-IDP has been shown to be very efficient in solving the multidrug scheduling optimization problem.
\end{abstract}

Index Terms-Drug scheduling (cancer) model, evolutionary algorithms (EAs), hybrid genetic algorithm-local search (GA-LS), iterative dynamic programming (IDP), memetic algorithms (MAs), optimal control.

\section{INTRODUCTION}

Cancer chemotherapy is one of the essential treatment methods for tumor therapy. One of the major aims of cancer chemotherapy is to minimize the number of tumor cells after a number of fixed treatment cycles. A cancer treatment schedule usually specifies a drug administration pattern that is repeated in cycles. The schedule of the drug administration determines the dosage of drugs to be infused into the patient's body. To minimize the tumor cells with minimum side effect after the end of treatment, it is crucial to design an optimal drug schedule for cancer chemotherapy. To find such an optimal treatment schedule, many mathematical models were developed to predict tumor growth when drugs were administered. There are many mathematical models introduced for cancer chemotherapy [9], [11], [20], [27], [31].

The use of combinations of drugs for cancer chemotherapy has proven to be more effective. Combination chemotherapy has several major advantages. Using multiple drugs for cancer chemotherapy can usually decrease the chance of drug resistance. The overall toxicity to the body or at least to an organ of the body can be reduced. A multiple chemotherapeutic drug model for cancer treatment subject to drug resistance was proposed by Martin and Teo [23] and Westman et al. [30].

According to Liang et al. [16], the toxicity equation of Martin's model [23] is not consistent with clinical experience and medical knowledge. Liang et al. modified the toxicity equation in Martin's single-drug model. In this correspondence, we have extended the twodrug model in [23] and modified the set of equations in [16] to model the responses of multidrug administrations. We use the multidrug model to simulate a set of clinical data with three drugs in our trails. The clinical data used in our simulations are collected by our oncol-

Manuscript received May 1, 2005; revised December 15, 2005, April 10, 2006, and June 12, 2006. This work was supported in part by the Hong Kong Special Administrative Region (SAR) under RGC Earmarked Grant 4173/04E and in part by the Chinese University of Hong Kong under RGC Research Grant Direct Allocation. This paper was recommended by Guest Editor Y. S. Ong.

S.-M. Tse, K.-S. Leung, K.-H. Lee, and T. S.-K. Mok are with the Department of Computer Science and Engineering, Chinese University of Hong Kong, Shatin, Hong Kong (e-mail: smtse@ cse.cuhk.edu.hk).

Y. Liang was with the Department of Computer Science and Engineering, Chinese University of Hong Kong, Shatin, Hong Kong. He is now with the Department of Computer Science and National Key Laboratory, Shantou University, Shantou 515063, China (e-mail: yliang@ @stu.edu.cn).

Digital Object Identifier 10.1109/TSMCB.2006.883265 ogist, who used three drugs throughout the course of cancer chemotherapy treatment. A multidrug model can help oncologists to design the best way to administer drugs for tumor treatments and save much effort for trial and error, especially when a new drug is discovered.

The objective of this study is to design or provide an efficient multidrug cancer chemotherapy schedule. We try to minimize the number of tumor cells after a fixed period of treatment. Our proposed model is formulated as an optimal control problem (OCP). The details of the problem statements will be presented in the following sections. It is usually very difficult to find the solutions to these OCPs due to the multimodal and highly nonlinear landscape of the search space. There are many optimization algorithms developed to solve OCPs [7], [16], [22], [24]. In this correspondence, we propose a new optimization algorithm to solve the multidrug scheduling optimization problem.

Exploration and exploitation are two major issues when designing a global search method. Exploration is important for the search algorithm to achieve global optimality, whereas exploitation searches around the neighborhood of good solutions to produce higher quality solutions. The search algorithm should strike a tactical balance between the two sometimes-conflicting goals. EAs are a class of global optimization algorithms proven very effective in solving a wide range of problems. However, traditional EAs may take a relatively longer time to converge to an optimum. Local search (LS) methods, based on different heuristics, can locally improve solution quality efficiently. These two common classes of optimization algorithms can be integrated to satisfy the two goals. Algorithms that hybridize nongenetic LSs to refine solution quality with EAs are called memetic algorithms (MAs). There are many studies on the design of MAs [2], [6], [12], [13], [25], [26].

Iterative dynamic programming (IDP), based on the idea of Bellman's dynamic programming (DP) [2], has been shown to effectively solve OCPs with very high dimension [17]-[21]. By integrating IDP, which is an efficient solution identification method, and an adaptive elitist genetic algorithm (AEGA), which is a global optimizer, we have created a new MA called MA-IDP which has been applied successfully to solve our proposed multidrug scheduling optimization problem.

This correspondence is organized as follows. Section II introduces the new drug administration dynamic model. The new MA designed to solve the dynamic model is presented in Section III. Section IV describes the implementation details and results of automating the optimal drug administrations with our proposed MA. Conclusions are drawn in Section V.

\section{New Drug Administration Dynamic Model}

Modeling of tumor responses for cancer chemotherapy with mathematical systems has a long history [10], [22], [29]. These mathematical models are widely used to predict the tumor responses and to optimize the control parameters and drug dosages infused in cancer chemotherapy. We model the problem as an OCP with a set of differential equations. The aim is to minimize the tumor size of the multidrug chemotherapy schedule under different constraints of drug resistances.

Our tumor growth model is a modified version of [23]. The original model proposed a two-drug model that considered the effects of drug resistance under the interactions between the tumor cells and the drugs administered. We extend this model by adding one more drug to the chemotherapy schedule. This means that our model will consider the interactions of three different drugs during the whole course of chemotherapy treatment. In fact, the model can be generalized to include even more drugs administered concurrently. 


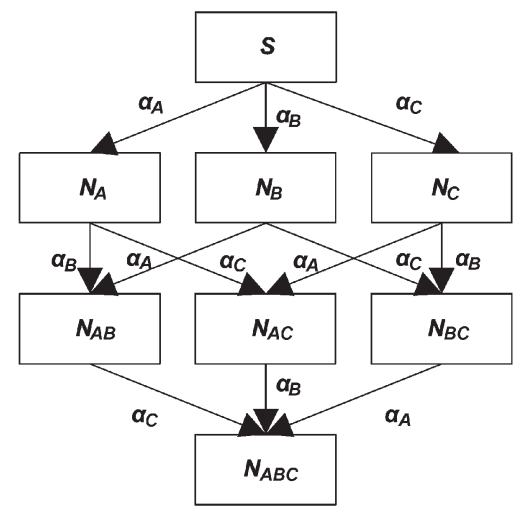

Fig. 1. Tumor growth under the action of three non-cross-resistant drugs. It consists of eight disjoint subpopulations, namely $S, N_{A}, N_{B}, N_{C}, N_{A B}$, $N_{A C}, N_{B C}$, and $N_{A B C}$. Mutations from sensitive phenotypes to resistant phenotypes are represented by arrows.

Using combinations of drugs for cancer chemotherapy has some advantages over a single-drug treatment since the chances of drug resistance are decreased [23]. The advantage of using multiple noncross-resistant drug therapy is that a greater variety of resistant subpopulations can be targeted. Also, simultaneous use of two or more drugs can kill more tumor cells if the toxic effects of these drugs on normal cells do not overlap significantly.

Our model is simulated with a set of clinical data collected by our oncologist, who used three drugs, namely: 1) irinotecan; 2) cisplatin; and 3) docetaxel, in the treatment cycles.

\section{A. Three-Drug Mathematical Model}

The model is developed by considering "pharmacokinetics." Pharmacokinetics is the mathematical study of what the body does during the course of absorption, distribution, metabolism, and excretion of drugs. In our model, a tumor comprises different compartments. A two-compartment model was proposed to study the effects of drugsensitive and drug-resistant tumor cells in [5] and [8], whereas a four-compartment model with two "cytotoxic drugs" for cancer chemotherapy was introduced in [1]. We develop a three-drug model in which the tumor contains eight compartments that are resistant to different drugs. In this section, all cells in our model are referring to tumor cells. Cells from sensitive compartments can transit to resistant compartments. Three chemotherapeutic drugs $A, B$, and $C$ are used for cancer treatment. Within a tumor, there are eight disjoint subpopulations. These subpopulations include the following: cells sensitive to all drugs, $S(t)$; cells resistant to exactly one drug, $\operatorname{drug} A$, drug $B$, and drug $C\left(N_{A}(t), N_{B}(t)\right.$, and $N_{C}(t)$, respectively); cells resistant to exactly two drugs, drugs $A$ and $B$, drugs $A$ and $C$, and drugs $B$ and $C\left(N_{A B}(t), N_{A C}(t)\right.$, and $N_{B C}(t)$, respectively); and the totally resistant cells for all drugs, $N_{A B C}(t)$. Cells from sensitive subpopulations may acquire resistance to drug $i$ "spontaneously" with probability $\alpha_{i}$. Multiple resistant cells arise from multiple mutations. Fig. 1 shows the transition of cells from sensitive compartments to resistant compartments. Since all patients enrolled in this study have advanced stage lung cancer, we can assume the same mutation rate for this group. In addition, the rate may be different in other cancer cell types or stages. The increase in the tumor population $N(t)$ at time $t$ per unit time due to cell proliferation is given by

$$
\frac{d N(t)}{d t}=\lambda N \quad \text { (exponential growth model) }
$$

where $\lambda$ is a positive constant representing the leading order exponential growth. Besides using the exponential growth model, we can also replace $N(t)$ with other growth models. Cells in all subpopulations share the same growth rate. Our model assumes that the effects of chemotherapy are instantaneous. This assumption is justifiable since the time unit we used, i.e., one day, is long enough for most treatment effects to happen.

The chemotherapeutic drugs are assumed to be non-cross-resistant since there are some classes of drugs that demonstrate no or little cross resistance. The mutation rates to a non-cross-resistant drug are the same for cells already resistant to another non-cross-resistant drug and cells from totally sensitive subpopulation. When the drugs are used simultaneously, it is assumed that the number of cells killed in each subpopulation is equal to the sum of cells killed when each drug is used individually. Drug $i$ is effective against its sensitive subpopulations only when the concentration $v_{i}$ is above the threshold concentration $v_{\mathrm{th}_{i}}$. A fraction of cells are killed by the chemotherapeutic drugs that are effective to them. For example, cells from subpopulation $N_{A B}$ with resistance to drugs $A$ and $B$ can only be killed with drug $C$, while no drug can eliminate cells from the totally resistant phenotype $N_{A B C}$. The fraction of cells killed by drug $i$ per unit time per drug concentration is denoted by $\kappa_{i}$. The set of dynamic equations modeling the description is shown in the following:

$$
\begin{aligned}
& \frac{d S}{d t}=\lambda\left[\left(1-\alpha_{A}-\alpha_{B}-\alpha_{C}\right) S\right] \\
& -\kappa_{A}\left(v_{A}-v_{\mathrm{th}_{A}}\right) H\left(v_{A}-v_{\mathrm{th}_{A}}\right) S \\
& -\kappa_{B}\left(v_{B}-v_{\mathrm{th}_{B}}\right) H\left(v_{B}-v_{\mathrm{th}_{B}}\right) S \\
& -\kappa_{C}\left(v_{C}-v_{\mathrm{th}_{C}}\right) H\left(v_{C}-v_{\mathrm{th}_{C}}\right) S \\
& \frac{d N_{A}}{d t}=\lambda\left[\left(1-\alpha_{B}-\alpha_{C}\right) N_{A}+\alpha_{A} S\right] \\
& -\left(\kappa_{B}\left(v_{B}-v_{\mathrm{th}_{B}}\right) H\left(v_{B}-v_{\mathrm{th}_{B}}\right)\right. \\
& \left.+\kappa_{C}\left(v_{C}-v_{\operatorname{th}_{C}}\right) H\left(v_{C}-v_{\operatorname{th}_{C}}\right)\right) N_{A} \\
& \frac{d N_{B}}{d t}=\lambda\left[\left(1-\alpha_{A}-\alpha_{C}\right) N_{B}+\alpha_{B} S\right] \\
& -\left(\kappa_{A}\left(v_{A}-v_{\mathrm{th}_{A}}\right) H\left(v_{A}-v_{\mathrm{th}_{A}}\right)\right. \\
& \left.+\kappa_{C}\left(v_{C}-v_{\operatorname{th}_{C}}\right) H\left(v_{C}-v_{\operatorname{th}_{C}}\right)\right) N_{B} \\
& \frac{d N_{C}}{d t}=\lambda\left[\left(1-\alpha_{A}-\alpha_{B}\right) N_{C}+\alpha_{C} S\right] \\
& -\left(\kappa_{A}\left(v_{A}-v_{\mathrm{th}_{A}}\right) H\left(v_{A}-v_{\mathrm{th}_{A}}\right)\right. \\
& \left.+\kappa_{B}\left(v_{B}-v_{\operatorname{th}_{B}}\right) H\left(v_{B}-v_{\operatorname{th}_{B}}\right)\right) N_{C} \\
& \frac{d N_{A B}}{d t}=\lambda\left[\left(1-\alpha_{A}\right) N_{A B}+\alpha_{B} N_{A}+\alpha_{A} N_{B}\right] \\
& -\kappa_{C}\left(v_{C}-v_{\mathrm{th}_{C}}\right) H\left(v_{C}-v_{\mathrm{th}_{C}}\right) N_{A B} \\
& \frac{d N_{A C}}{d t}=\lambda\left[\left(1-\alpha_{B}\right) N_{A C}+\alpha_{C} N_{A}+\alpha_{A} N_{C}\right] \\
& -\kappa_{B}\left(v_{B}-v_{\mathrm{th}_{B}}\right) H\left(v_{B}-v_{\mathrm{th}_{B}}\right) N_{A C} \\
& \frac{d N_{B C}}{d t}=\lambda\left[\left(1-\alpha_{A}\right) N_{B C}+\alpha_{B} N_{C}+\alpha_{C} N_{B}\right] \\
& -\kappa_{A}\left(v_{A}-v_{\mathrm{th}_{A}}\right) H\left(v_{A}-v_{\mathrm{th}_{A}}\right) N_{B C} \\
& \frac{d N_{A B C}}{d t}=\lambda\left[\alpha_{A} N_{B C}+\alpha_{C} N_{A C}+\alpha_{C} N_{A B}\right]
\end{aligned}
$$

where $H(x)$ is the Heaviside step function defined as

$$
H(x)= \begin{cases}1, & \text { if } x \geq 0 \\ 0, & \text { otherwise }\end{cases}
$$


TABLE I

Summary of PARAmeters of THE MultidRug MOdel

\begin{tabular}{cl}
\hline \hline Parameter & \multicolumn{1}{c}{ Description } \\
\hline$\kappa_{i}$ & $\begin{array}{l}\text { proportion of cells killed by drug } i \text { per unit time per drug } \\
\text { concentration }\end{array}$ \\
$\eta_{i}$ & constant related to metabolic rate of drug $i$ \\
$\gamma_{i}$ & decay constant of drug $i$ \\
$\alpha_{i}$ & mutation rate from sensitive subpopulation to resistant \\
& subpopulation of drug $i$ \\
$\lambda$ & constant related to the growth rate \\
$v_{t h_{i}}$ & threshold concentration of drug $i$ to be effective \\
\hline \hline
\end{tabular}

The initial cell subpopulations are as follows:

$$
\begin{aligned}
S(0) & =S_{0} \quad N_{A}(0)=N_{A_{0}} \quad N_{B}(0)=N_{B_{0}} \\
N_{C}(0) & =N_{C_{0}} \quad N_{A B}(0)=N_{A B_{0}} \quad N_{A C}(0)=N_{A C_{0}} \\
N_{B C}(0) & =N_{B C_{0}} \quad N_{A B C}(0)=N_{A B C_{0}} .
\end{aligned}
$$

Besides studying tumor population, we also need to study drug concentrations and drug toxicities. We adopt the equations of drug concentrations and toxicities from [16].

The concentration of each drug is assumed to decay exponentially. Equations (9)-(11) describe the change of drug concentrations inside the patient's body. The rate to deliver drug $i$ is denoted by the variable $u_{i}$, where the concentration of drug $i$ decays with half-life $\gamma_{i}$, which depends on the biochemical property of the drug, i.e.,

$$
\begin{aligned}
& \frac{d v_{A}}{d t}=u_{A}-\gamma_{A} v_{A} \\
& \frac{d v_{B}}{d t}=u_{B}-\gamma_{B} v_{B} \\
& \frac{d v_{C}}{d t}=u_{C}-\gamma_{C} v_{C} .
\end{aligned}
$$

The cumulative toxicity can be measured by the cumulative drug concentration within the patient's body, which affects the population of the immune cells. It is actually an index reflected by the normal bone marrow cell count. In our model, we simplified it as a function of drug concentrations and metabolic rate. The relationship between the cumulative drug toxicity and the drug concentration inside the body is described in (12)-(14). The cumulative drug toxicity $\tau_{i}$ increases with the concentration of drug $i$ (i.e., $v_{i}$ ) and decreases with the rate of metabolism of drug $i$ inside the body. The rate of metabolism of drug $i$ inside the body is assumed to be directly proportional to the cumulative drug toxicity $\tau_{i}$ with a proportion constant $\eta_{i}$, i.e.,

$$
\begin{aligned}
\frac{d \tau_{A}}{d t} & =v_{A}-\eta_{A} \tau_{A} \\
\frac{d \tau_{B}}{d t} & =v_{B}-\eta_{B} \tau_{B} \\
\frac{d \tau_{C}}{d t} & =v_{C}-\eta_{C} \tau_{C} .
\end{aligned}
$$

Parameters of the proposed multidrug model are shown in Table I.

\section{B. $O C P$}

After we have developed a mathematical model to describe the behavior of tumor subpopulations throughout the course of treatment, we want to determine the optimal treatment regime during the course of therapy. Based on the set of dynamic equations, we can formulate our problem as an OCP for optimization. Numerous research [4], [9], [28] shows that optimal control theory can be applied to identify
TABLE II

Summary of State Variables of the Multidrug Model

\begin{tabular}{clc}
\hline \hline Parameter & \multicolumn{1}{c}{ Description } & Unit \\
\hline$S$ & totally sensitive subpopulation & - \\
$N_{i}$ & subpopulation resistant to drug $i$ & - \\
$N_{i j}$ & subpopulation resistant to drugs $i$ and $j$ & - \\
$N_{i j k}$ & subpopulation resistant to drugs $i, j$ and $k$ & - \\
$v_{i}$ & concentration of drug $i$ in the cancer site & D \\
$\tau_{i}$ & toxicity dues to drug $i$ in the body & day D \\
\hline \hline
\end{tabular}

TABLE III

Summary of Control Variables of the Multidrug Model

\begin{tabular}{clc}
\hline \hline Parameter & \multicolumn{1}{c}{ Description } & Unit \\
\hline$u_{A}(t)$ & deliver rate of drug $A$ at a specific time, $t$ & $\mathrm{D}$ \\
$u_{\mathrm{B}}(t)$ & deliver rate of drug $B$ at a specific time, $t$ & $\mathrm{D}$ \\
$u_{C}(t)$ & deliver rate of drug $C$ at a specific time, $t$ & $\mathrm{D}$ \\
\hline \hline
\end{tabular}

the optimal chemotherapeutic schedule. The general form of an OCP consists of a dynamic system that describes a set of state variables, i.e.,

$$
\begin{aligned}
& \mathbf{x}(t)=\mathbf{f}(\mathbf{x}(t), \mathbf{u}(t), t) \\
& \mathbf{x}(0)=\mathbf{x}_{0}
\end{aligned}
$$

where $\mathbf{x}(t)$ is an $(n \times 1)$ state variable vector and $\mathbf{u}(t)$ is an $(m \times 1)$ control variable vector bounded by

$$
\alpha_{j} \leq u_{j}(t) \leq \beta_{j}, \quad j=1,2, \ldots, m .
$$

Unlike those localized treatment methods such as surgery or radiotherapy, actions taken by chemotherapeutic drugs are effective not only to the tumor site but also to all organs of the body. Serious adverse effects will arise when the concentration or toxicity of a drug inside the body exceeded a certain threshold. The level of drug concentration and toxicity should be kept below a tolerable level throughout the course of treatment. Thus, we imposed a set of constraints on drug concentration and toxicity to our model to ensure that the patient can tolerate the toxic side effects of the drug. These constraints are as follows:

$$
\begin{array}{ll}
v_{i} \leq 50, & i \in\{A, B, C\} \\
\tau_{i} \leq 100, & i \in\{A, B, C\} .
\end{array}
$$

Since our goal is to minimize the tumor size after the course of chemotherapy, we have our performance index $I$ as

$$
I=S\left(t_{f}\right)+\sum_{i} N_{i}\left(t_{f}\right)+\sum_{i, j} N_{i j}\left(t_{f}\right)+\sum_{i, j, k} N_{i j k}\left(t_{f}\right)
$$

where $i, j, k \in\{A, B, C\}, i \neq j \neq k$, and $t_{f}=84$ days (which is the length of the treatment cycles).

The state variables and control variables of our model are listed in Tables II and III, respectively.

\section{Proposed Memetic Optimization Algorithm}

Optimization plays a key role in the fields of artificial intelligence, computer science, and operational research. We propose a new memetic optimization algorithm (MA-IDP) by hybridizing a population-based search (AEGA) with an LS (IDP). AEGA [15] determines the dissimilarity among individuals by the relative ascending (or descending) directions in maximization (minimization) problems and a distance threshold parameter $\sigma_{s}$. Based on the dissimilarity between individuals, AEGA can eliminate redundant individuals that share the same peak and create elitist individuals on the unexplored peaks. 


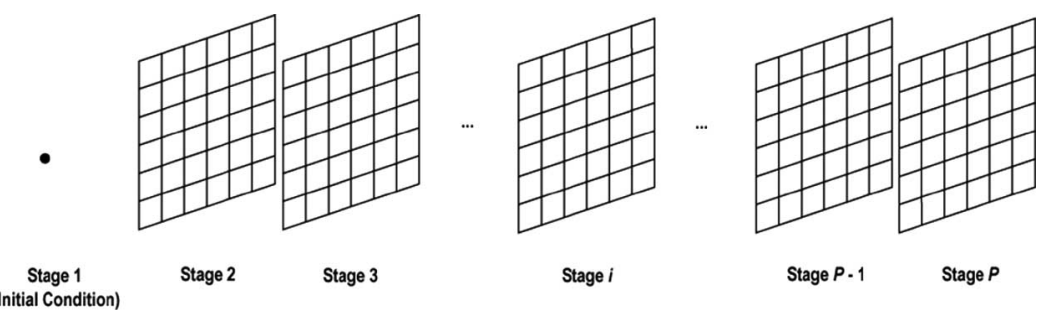

Fig. 2. Construction of state grid points over all stages. Stage 1 only has the initial condition of the state grid point, and each state grid point in each stage represents a set of respective values of the state variables.

IDP [20] divides the problem into different time stages and improves the solution iteratively. This section briefly reviews IDP and AEGA, the details of the MA-IDP, and how we apply MA-IDP to solve our drug schedule optimization problem.

\section{A. IDP}

IDP is an LS method proposed by Luus et al. [19]. The fundamental ideas of IDP are based on DP [3]. Applications of IDP to optimize many real-world OCPs were explored in numerous papers [17]-[21]. The general formulation of an OCP has been discussed in the previous section. The algorithm of IDP includes the following steps.

Division of Time Interval: To apply IDP for optimization, we have to construct a sequence of time stages for the problem as required by DP. We divide the time interval into $P$ stages, each of length $L$, such that

$$
L=\frac{t_{f}}{P}
$$

In our problem, $t_{f}$ is the length of the treatment schedule (84 days).

Construction of State Grid Points: A set of values taken by a state vector $\mathbf{x}(n \times 1)$ is represented as a grid point. For the state vector $\mathbf{x}$ at the first stage, it takes the value of the specified initial condition. For each variable $x_{i}$ of the state vector $\mathbf{x}$ at the remaining stages, it is allowed to take $N$ values over its variable domain at that time stage. This means that there are $N^{n}$ grid points for stages $2,3, \ldots, P$. Fig. 2 shows the construction of state grids over all stages. The best value of $\mathbf{x}$ obtained from the previous iteration is set as the central point of the grid.

Construction of Control Grid Points: For each variable $u_{i}$ of the control vector $\mathbf{u}(m \times 1)$ at each stage, it is allowed to take $M$ values over its variable domain at that time stage. This means that there are $M^{m}$ grid points for each stage. The variable domain of the control vector contracts during iteration. The central value of the variable domain is taken as the best value of $\mathbf{u}$ from the previous iteration.

Iterative Procedure: The iteration procedure starts from the last stage $P$. It evaluates the performance index of each state grid point $\mathbf{x}$ by integrating (1)-(14) from time $t_{f}-L$ to $t_{f}$ with its $M^{m}$ control vector values $\mathbf{u}$.

The best control vector of a particular state vector is the one that gives the minimum value of the performance index among these $M^{m}$ control vector values.

After obtaining the best control vector at stage $P$, it steps backward to stage $P-1$, which corresponds to the time interval $t_{f}-2 L \leq$ $t<t_{f}-L$. Similarly for stage $P$, we consider $M^{m}$ control values of each state grid point. After we have evaluated (19) from $t_{f}-2 L$ to $t_{f}-L$, the state vector $\mathbf{x}\left(t_{f}-L\right)$ may not match any state vector of the next stage (stage $P$ ). To continue to integrate the equations to the next stage, we use the optimal control vector of the grid point in the next stage that is closest to the state $\mathbf{x}\left(t_{f}-L\right)$. Fig. 3 shows the details on how to continue the integration to the next stage, with $n=2$, $N=7, m=1$, and $M=5$. For example, for the grid point $(3,2)$ in

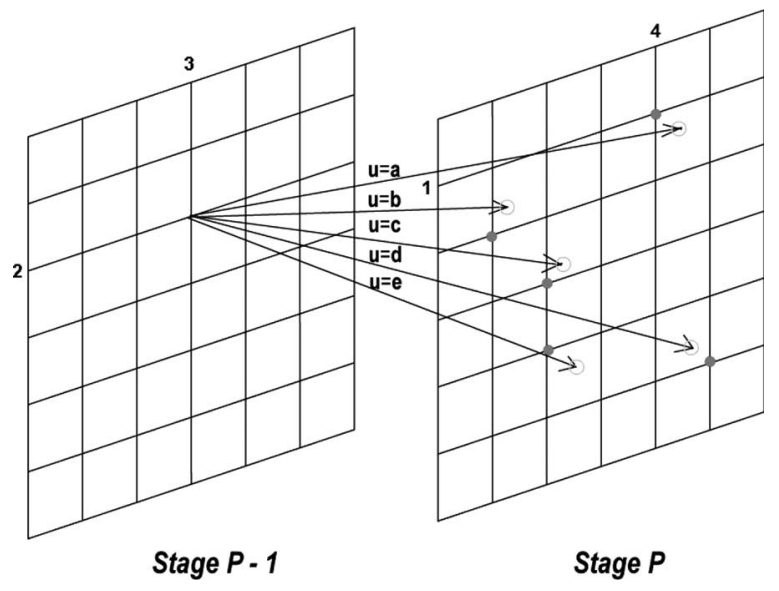

Fig. 3. Details on how to continue the integration with the optimal control of the closest grid point at the next stage. At stage $P-1$, based on the performance of the closest grid point at stage $P$ obtained in the previous step, the best set of control values $\mathbf{u}$ for the grid point $(2,3)$ will be used to continue the integration to the last stage. For example, if $\mathbf{u}=\mathbf{a}$ is the best values for grid point $(2,3)$ at stage $P-1$, the best values of its closest grid point $(1,4)$ at stage $P$ will be used to continue the integration to the last stage.

stage $P-1$ with $\mathbf{u}=\mathbf{a}$, the state grid point $(4,1)$ is the closest grid point among the $(7 \times 7)$ state grid points in stage $P$. Therefore, we use the optimal control vector $\mathbf{u}_{\text {opt }}$ of the grid point $(4,1)$ in stage $P$ obtained from the previous step to continue the integration to the final time stage. We calculate the performance index of the grid point $(3,2)$ in stage $P-1$ with the remaining control vectors. The optimal control for that grid point is the control vector that gives the minimum value of the performance index. The integration procedure is continued in a backward manner from stage $P-2$ to stage 1 .

Systematic Reduction in Region Size: The optimal control policy from the previous iteration provides the central values of the control vectors for each stage, while the optimal trajectory from the previous iteration updates the central grid point of each stage. The search ranges $s_{i}$ and $r_{j}$, which correspond to the control and state vectors at each stage, respectively, are contracted by a region contraction factor $\gamma$ for finer resolution. Thus, the search region for $x_{i}$ and $u_{j}$ is $x_{i}^{*} \pm s_{i}$ and $u_{j}^{*} \pm r_{j}$, respectively, where $x_{i}^{*}$ and $u_{j}^{*}$ are the optimal trajectory of the $i$ th state variable and the optimal control policy of the $j$ th control variable at that stage, respectively. If the search region is outside the domain of the variable, it is substituted with the value of the upper or lower bound. The whole iteration procedure is repeated until the optimal control policy is obtained with sufficient accuracy. The IDP algorithm can locate an optimal solution quickly, but there is no guarantee that the optimum found is the global one.

\section{B. $A E G A$}

We regard two individuals as similar if they share the same bangbang control patterns (same cycle length) in all three pairs of their 


\begin{tabular}{|c||c||c|c|c|}
\hline \multirow{1}{*}{ Drug } & \multirow{2}{*}{ Head } & \multicolumn{3}{c||}{ Bang-bang Control Cycles } \\
\cline { 3 - 4 } 1 & Dosages & Starting Day & Dosage & Cycle Length \\
\hline \hline Drug & Head & \multicolumn{3}{|c|}{ Bang-bang Control Cycles } \\
\cline { 3 - 4 } 2 & Dosages & Starting Day & Dosage & Cycle Length \\
\hline \hline Drug & Head & \multicolumn{3}{|c|}{ Bang-bang Control Cycles } \\
\cline { 3 - 4 } 3 & Dosages & Starting Day & Dosage & Cycle Length \\
\cline { 3 - 5 } & & &
\end{tabular}

Fig. 4. Chromosome that encodes a drug administration schedule.

schedules. Otherwise, the two individuals are considered to be dissimilar. The elitist of similar individuals is conserved, while the redundant ones are eliminated from the population by the genetic operators during iteration. With the aids of the elitist genetic operators, the algorithm can adaptively adjust the population size according to the features of the problems. It minimizes the number of individual to explore each optimum and search the landscape optima in parallel. We will show the details of the bang-bang control and our elitist genetic operators in the coming sections.

\section{Proposed MA}

We apply the efficient IDP algorithm as a genetic operator of the multimodal GA (AEGA) [15]. We also employ IDP to initialize our candidate solutions. The advantages of using IDP are threefold: IDP can refine the solution quality and repair a solution that violates any constraint, while AEGA can find global and multiple solutions for the problem. In clinical treatment, an oncologist expects to select different drug scheduling policies for different patients. Our MA-IDP can obtain multiple solutions and identify multiple efficient drug scheduling policies for a doctor to design the treatment method depending on various patient conditions.

\section{Bang-Bang Control}

Cancer chemotherapy treatments usually consist of a number of cycles. The dosage of drugs infused to the patient is fixed in each cycle. To find such kind of drug administration patterns, bang-bang solutions [14] are used. Solution schedule is a schedule that infuses either a fixed or zero dosage to the patient's body for each day of treatment cycles. Bang-bang control patterns are applied to the schedule to push toward the constraint limits.

\section{E. Chromosome Encoding}

We encode a chromosome of our problem as a schedule of drug dosages throughout the treatment cycles. It consists of the dosage of each drug (as a real number) throughout the course of the 84-day chemotherapy treatment. Fig. 4 shows a chromosome of the drug schedule. The chromosome comprises two parts, i.e., 1) the head dosages and 2) the tail bang-bang controls (in cycles). The head dosages are the drug dosages for the early days of the treatment. For the remaining days, a cyclewise representation is used to encode the bang-bang control cycles, which consist of three numbers, i.e., 1) the starting day; 2) the dosage; and 3 ) the cycle length. For example, the chromosome $(50,40,30) \mid(4,35,3)$ represents the drug schedule of $[50,40,30 \mid 35,0,0,35,0,0, \ldots, 35,0,0]$. With this cyclewise representation, we can generate a bang-bang solution for the drug schedule accurately and easily. The valid range for each gene of the chromosome is from 0 to 150 . Bang-bang solutions are generated first by the one-iteration + one-stage IDP followed by the generation of the head dosages by the one-iteration +84 -stage IDP. Fig. 5 illustrates example schedules for obtaining the bang-bang solutions. Example schedules after optimizing the head dosages are shown in Fig. 6.
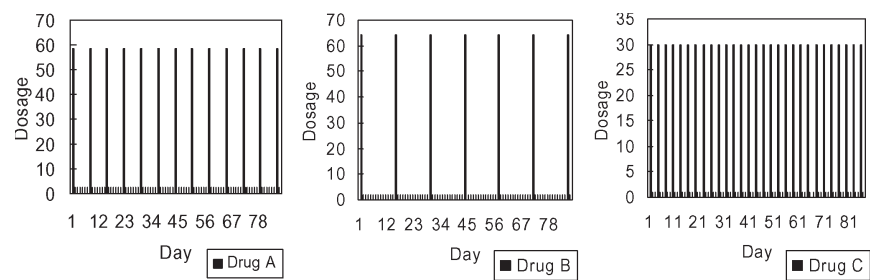

Fig. 5. Example schedules after applying one-iteration + one-stage IDP.
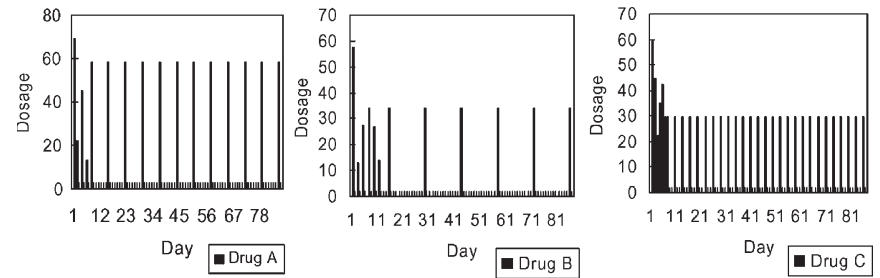

Fig. 6. Example schedules after applying one-iteration + day-stage IDP.

\section{F. Initialize Schedule}

We apply IDP with a single iteration and one time stage (oneiteration + one-stage IDP) to initialize our candidate schedules such that a fixed dosage of drugs is administered after a certain time interval. A bang-bang control schedule pattern is obtained. Fig. 5 shows an example of our initialized schedule, a schedule of $\operatorname{drug} A$ with time interval equals to seven days, a schedule of drug $B$ with time interval equals to 14 days, and a schedule of $\operatorname{drug} C$ with time interval equals to three days. The dosage of each drug is fixed within each schedule.

From our observations, a bang-bang control solution obtained after initialization can push toward the constraint limits in the later phases of the treatment cycles, while the schedule still allows us to infuse higher dosage of drug in the early phases. After initialization, our algorithm will try to push toward the limit in the early phases by increasing the drug dosages. We improve the schedule by trying higher drug dosage in the early phases of a schedule with IDP. We applied IDP with a single iteration and day time stages (one-iteration + day-stage IDP) ${ }^{1}$ to initialize our candidate schedules, where day is the number of days in the treatment period. Fig. 6 shows the example schedules after applying one-iteration +84 -stage IDP.

In the first few days of the treatment period, it is suggested to kill as many tumor cells as possible since drug resistances are not acquired in the patient's body. Drug dosages are adjusted by IDP in the first few treatment days. When the patient gradually acquires drug resistances, a fixed cycle of fixed patterns is used, corresponding to the bang-bang control pattern of our schedule in the later treatment days.

\section{G. Elitist Crossover}

Crossover is performed by exchanging a pair of complete schedule of a single drug of two chromosomes. Two chromosomes are selected randomly from the population as parents. If the two individuals share the same bang-bang control patterns (same cycle length) for all drug schedules, we consider the two schedules are similar. If two schedules are similar, the one with lower fitness will be eliminated. Otherwise, crossover will be performed to generate two offspring. Fig. 7 shows the details of the crossover operator. It exchanges the drug schedules between two chromosomes, and one-iteration + 84-stage IDP is then applied to each offspring.

${ }^{1}$ IDP with only one pass in its iterative procedure and the time interval is divided into day time stages ( 84 time stages in our problem). 


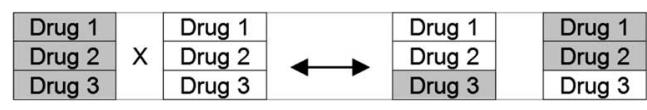

Fig. 7. Crossover operator that exchanges the drug schedules between two chromosomes.

\begin{tabular}{|l|l|}
\hline Drug 1 \\
\cline { 1 - 1 } Drug 2 \\
\cline { 1 - 1 } Drug 3 & $\longrightarrow$ Drug 1 \\
\cline { 1 - 1 } & Drug 2 \\
\hline Drug 3 \\
\hline
\end{tabular}

Fig. 8. Mutation operator that changes a drug schedule of a chromosome.

After applying the crossover operator, we evaluate the fitness of the two offspring and then select the two individuals with higher fitness among the parents and offspring for the next generation.

\section{H. Elitist Mutation}

Mutation operator randomly changes the dosage of a drug schedule in the way similar to the initialization step. Fig. 8 depicts the mutation operation. If the mutated schedule has a similar one in the population, the one with lower fitness will be eliminated. Otherwise, both parent and offspring are carried to the next generation.

\section{Fitness Evaluation}

The fitness of each candidate solution to be maximized is defined as $10^{4} / N\left(t_{f}\right)$, where $N$ is the sum of the numbers of tumor cells of all subpopulations.

\section{J. Algorithm Outline}

We apply IDP within the genetic operators of AEGA to explore multiple high-quality solutions of the drug model efficiently. The outline of MA-IDP is as follows.

1) Set $t=0$ and initialize a chromosome population $P(t)$ (bangbang solutions based on one-iteration + one-stage IDP).

2) Optimize and evaluate the fitness of $P(t)$ by using oneiteration + day-stage IDP.

3) While (termination condition not satisfied) Do

a) Elitist crossover operation to generate $P(t+1)$.

i) Check the dissimilarity of the randomly selected parents $p_{i}$ and $p_{j}$.

ii) If the parents $p_{i}$ and $p_{j}$ are similar, the elitist crossover operation conserves the better one of them to $P(t+1)$.

Else, perform crossover operation to generate two offspring $c_{i}$ and $c_{j}$ and optimize and evaluate the fitness of $c_{i}$ and $c$ by using one-iteration + day-stage IDP; then, select the better two among the parents and their offspring into $P(t+1)$.

b) Elitist mutation operation to generate $P(t+1)$.

i) Perform the mutation operation to generate the offspring $c_{i}$ from the parent $p_{i}$.

ii) Optimize and evaluate the fitness of $c_{i}$ by using oneiteration + day-stage IDP.

iii) If $c_{j}$ is dissimilar with any individual in $P(t)$, the elitist mutation operation conserves $p_{i}$ and $c_{i}$ together to $P(t+1)$.

Else, select the better one of similar individuals into $P(t+1)$.

4) Stop if the termination condition is satisfied; otherwise, $t \leftarrow t+$ 1 and go to Step 3 ).
TABLE IV

PARAMETER Setting OF THe TUMor Model

\begin{tabular}{ccc|ccc}
\hline \hline Parameter & Value & Unit & Parameter & Value & Unit \\
\hline$\eta_{A}$ & 0.4 & day $^{-1}$ & $S_{0}$ & $\begin{array}{c}4.60517 \\
\times 10^{11}\end{array}$ & - \\
$\eta_{B}$ & 0.5 & day $^{-1}$ & $N_{A_{0}}$ & 0 & - \\
$\eta_{C}$ & 0.45 & day $^{-1}$ & $N_{B_{0}}$ & 0 & - \\
$\alpha_{A}$ & 0.008 & - & $N_{C_{0}}$ & 0 & - \\
$\alpha_{B}$ & 0.01 & - & $N_{A B_{0}}$ & 0 & - \\
$\alpha_{C}$ & 0.014 & - & $N_{A C_{0}}$ & 0 & - \\
$\gamma_{A}$ & 0.32 & day $^{-1}$ & $N_{B C_{0}}$ & 0 & - \\
$\gamma_{B}$ & 0.27 & day $^{-1}$ & $N_{A B C_{0}}$ & 0 & - \\
$\gamma_{C}$ & 0.25 & day $^{-1}$ & $\kappa_{A}$ & 0.0084 & day $^{-2} \mathrm{D}^{-1}$ \\
$v_{t h_{A}}$ & 10.0 & day D & $\kappa_{B}$ & 0.0074 & day $^{-2} \mathrm{D}^{-1}$ \\
$v_{t h_{B}}$ & 10.0 & day D & $\kappa_{C}$ & 0.0092 & day $^{-2} \mathrm{D}^{-1}$ \\
$v_{t h_{C}}$ & 10.0 & day D & $\lambda$ & 0.0099 & day $^{-1}$ \\
\hline \hline
\end{tabular}

TABLE $\mathrm{V}$

EXPERIMENTAL SETTINGS FOR IDP, MA-IDP, AND AEGA

\begin{tabular}{c|c|c|c}
\hline \hline & \multicolumn{3}{|c}{ Algorithms } \\
\cline { 2 - 4 } & MA-IDP & AEGA & IDP \\
\hline Initial Population Size & 10 & 10 & - \\
No. of Generations (to converge) & 50 & 5000 & - \\
Crossover Probability & 1.0 & 1.0 & - \\
Mutation Probability & 1.0 & 1.0 & - \\
Number of State Grid Points & 4 & - & 4 \\
Number of Control Grid Points & 10 & - & 10 \\
Region contraction factor, $\gamma$ & 0.99 & - & 0.99 \\
\hline \hline
\end{tabular}

\section{EXPERIMENTS AND RESULTS}

We have applied our proposed MA to find high-quality schedules for our proposed model presented in Section III.

\section{A. Experimental Settings}

We have carried out a number of experiments with MA-IDP and compared the results with AEGA and IDP. The initial population sizes for MA-IDP and AEGA were both set to 10. The population sizes changed dynamically. We set the crossover and mutation probabilities to 1.0. Both algorithms were iterated until they converged. For MA-IDP and IDP, the numbers of state grid points and control grid points were set to 4 and 10, respectively. The values of the parameters of the model are presented in Table IV. From day 1 to day 84, all three drugs, i.e., $A, B$, and $C$, are administered simultaneously. To achieve higher accuracy, the drug dynamic model was simulated using the fourth-order Runge-Kutta numerical integration method with a step size of 0.01 day. Table $\mathrm{V}$ depicts the experimental settings for MA-IDP, AEGA, and IDP. The number of generations is set just large enough to ensure that the population will converge in both the MA-IDP and AEGA experiments. Based on our experimental settings, MA-IDP and AEGA converged around the 50th and 5000th generations, respectively. The population size is changed dynamically by the genetic operators.

\section{B. Optimization Results}

We have automated the multidrug scheduling model with our proposed algorithm, i.e., MA-IDP, and 12 multiple solutions were obtained. Fig. 9 shows the details of each state variable of the best schedule obtained (schedule 1). From the plots, we can see that the schedule tries to push toward the toxicity limits of each drug 


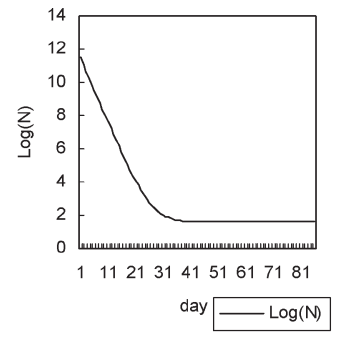

(a)

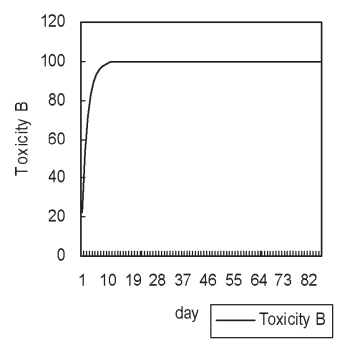

(f)

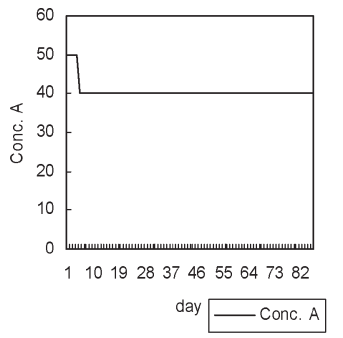

(b)

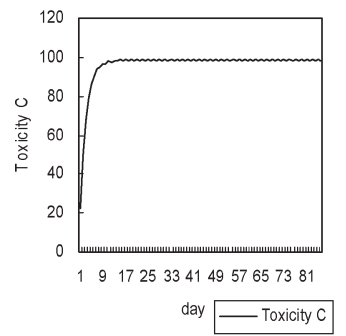

(g)

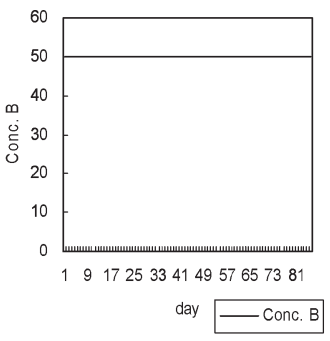

(c)

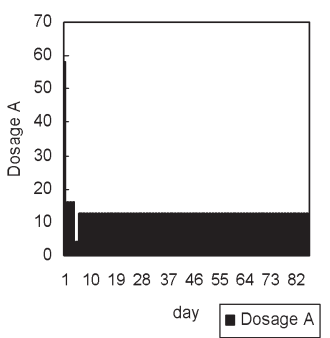

(h)

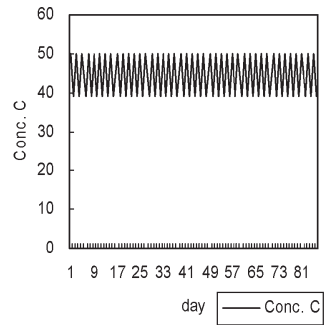

(d)

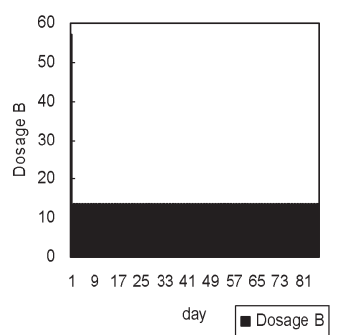

(i)

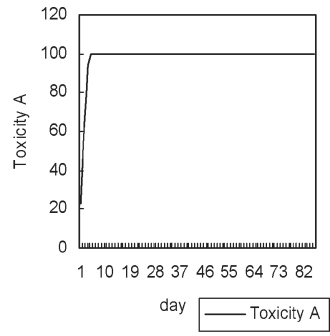

(e)

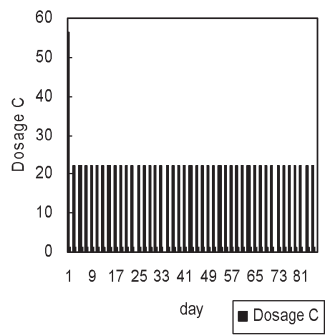

(j)

Fig. 9. Optimization result. (a) Logarithm of the number of tumor cells. (b) Concentration of drug $A$ at the cancer site. (c) Concentration of drug $B$ at the cancer site. (d) Concentration of drug $C$ at the cancer site. (e) Toxicity of drug $A$ inside the body. (f) Toxicity of drug $B$ inside the body. (g) Toxicity of drug $C$ inside the body. (h) Administration schedule of drug $A$ (dosage unit: D). (i) Administration schedule of drug $B$ (dosage unit: D). (j) Administration schedule of drug $C$ (dosage unit: D).

TABLE VI

COMPARISON BETWEEN DifFERENT ALgORITHMS

\begin{tabular}{c|c|c|c}
\hline \hline & \multicolumn{3}{|c}{ Algorithms } \\
\cline { 2 - 4 } & MA-IDP & AEGA & IDP \\
\hline Average Fitness & $\mathbf{3 7 8 . 5 7}$ & 270.23 & 355.00 \\
Best Fitness & $\mathbf{4 2 6 . 9 0}$ & 413.86 & 376.86 \\
Standard Deviation (Fitness) & 43.47 & 87.03 & $\mathbf{2 9 . 2 5}$ \\
Average Runtime (sec.) & 49.74 & 1474.53 & $\mathbf{2 9 . 3 0}$ \\
Multiple Solutions & Yes & Yes & No \\
\hline \hline
\end{tabular}

[Fig. 9(e)-(g)]. The tumor cell count is reduced exponentially during the early phases of the treatment period. Due to drug resistances, the tumor population gradually converges to a small number. It is envisaged that the immune system could seek out and eliminate these small tumors in the body.

The experiments with the three algorithms (i.e., MA-IDP, AEGA, and IDP) were repeated ten times, and the results are listed in Table VI. We can see that MA-IDP outperformed both AEGA and IDP in terms of the average and the best fitness (of the best individual in each run). MA-IDP has also preserved the ability of AEGA to find multiple solutions in a run. Although the average runtime and convergence power (standard deviation) for IDP is better than MA-IDP, the values are still comparable. It demonstrates that MA-IDP has combined the high convergence power of IDP and the strong global optimization ability of AEGA. Fig. 9(a) shows the high quality of the solutions, and the tumor size can be reduced effectively in the early stage of the treatment. The solution schedules [Fig. 9(h)-(j)] can provide the oncologist with insights for designing their treatment schedules and patterns.

\section{CONCLUSION AND FUture WORK}

This correspondence has proposed an optimal control tumor model for multidrug cancer chemotherapy schedule optimization with the help of an oncologist. We have integrated the AEGA and IDP algorithms to form a new MA (i.e., MA-IDP).

Our model can automate the trial and error procedure to suggest the ways to administer different drugs, especially for newly discovered drugs. We plan to tune our multidrug model parameters with multidrug clinical data and simulate biochemical behaviors of the drugs in the future work. We will then study a variety of drug combinations, doses, and treatment patterns with different schedules.

\section{ACKNOWLEDGMENT}

The authors would like to thank Y. S. Ong and the four anonymous reviewers for their constructive comments that have helped greatly in improving this correspondence.

\section{REFERENCES}

[1] M. Abundo and C. Rossi, "Numerical simulation of a stochastic model for cancerous cells submitted to chemotherapy," J. Math. Biol., vol. 27, no. 1, pp. 81-90, 1989.

[2] N. K. Bambha, S. S. Bhattacharyya, J. Teich, and E. Zitzler, "Systematic integration of parameterized local search into evolutionary algorithms," IEEE Trans. Evol. Comput., vol. 8, no. 2, pp. 137-154, Apr. 2004.

[3] R. E. Bellman, Dynamic Programming. Princeton, NJ: Princeton Univ. Press, 1957.

[4] Y. Biran and B. McInnis, "Optimal control of bilinear systems: Timevarying effects of cancer drugs," Automatica, vol. 15, no. 3, pp. 325-329, May 1979.

[5] J. L. Boldrini and M. I. S. Costa, "Therapy burden, drug resistance, and optimal treatment regimen for cancer chemotherapy," IMA J. Math. Appl. Med. Biol., vol. 17, no. 1, pp. 33-51, Mar. 2000.

[6] E. K. Burke, G. Kendall, and E. Soubeiga, "A tabu-search hyperheuristic for timetabling and rostering," J. Heuristics, vol. 9, no. 6, pp. 451-470, 2003.

[7] E. F. Carrasco and J. R. Banga, "Dynamic optimization of batch reactors using adaptive stochastic algorithms," Ind. Eng. Chem. Res., vol. 36, no. 6, pp. 2252-2261, 1997.

[8] M. I. S. Costa, J. L. Boldrini, and R. C. Bassanezi, "Drug kinetics and drug resistance in optimal chemotherapy," Math. Biosci., vol. 125, no. 2, pp. 191-209, Feb. 1995.

[9] L. G. de Pillis and A. E. Radunskaya, "A mathematical tumor model with immune resistance and drug therapy: An optimal control approach," Theor. Med., vol. 3, pp. 79-100, 2001.

[10] M. Eisen, Mathematical Models in Cell Biology and Cancer Chemotherapy, Lecture Notes in Bioinformatics. New York: Springer-Verlag, 1979.

[11] S. N. Gardner, "Cell cycle phase-specific chemotherapy: Computation methods for guiding treatment," Cell Cycle, vol. 1, no. 6, pp. 369-374, Nov./Dec. 2002. 
[12] H. Ishibuchi, T. Yoshida, and T. Murata, "Balance between genetic search and local search in memetic algorithms for multiobjective permutation flowshop scheduling," IEEE Trans. Evol. Comput., vol. 7, no. 2, pp. 204-223, Apr. 2003.

[13] N. Krasnogor and J. E. Smith, "A tutorial for competent memetic algorithms: Model, taxonomy and design issues," IEEE Trans. Evol. Comput., vol. 9, no. 5, pp. 474-488, Oct. 2005.

[14] U. Ledzewicz and Schãttler, "Optimal bang-bang controls for a twocompartment model in cancer chemotherapy," J. Optim. Theory Appl., vol. 114, no. 3, pp. 609-637, Sep. 2002.

[15] K.-S. Leung and Y. Liang, "Adaptive elitist-population based genetic algorithm for multimodal function optimization," in Proc. GECCO, 2003, pp. 1160-1171.

[16] Y. Liang, K.-S. Leung, and T. S.-K. Mok, "A novel evolutionary drug scheduling model in cancer chemotherapy," IEEE Trans. Inf. Technol. Biomed., vol. 10, no. 2, pp. 237-245, 2006.

[17] R. Luus and B. Bojkov, "Global optimization of the bifunctional catalyst problem," Can. J. Chem. Eng., vol. 72, pp. 160-163, 1994.

[18] R. Luus, J. Dittrich, and F. J. Keil, "Multiplicity of solutions in the optimization of a bifunctional catalyst blend in a tubular reactor," Can. J. Chem. Eng., vol. 70, no. 4, pp. 780-785, 1992.

[19] R. Luus, F. Hartig, and F. Keil, "Optimal control of batch reactors by iterative dynamic programming," J. Process Control, vol. 4, no. 4, p. 218, 1994.

[20] R. Luus, "Iterative dynamic programming," in Monographs and Surveys in Pure and Applied Mathematics. London, U.K.: Chapman \& Hall, 2000.

[21] — - "Optimal control by dynamic programming using accessible grid points and region reduction," Hung. J. Ind. Chem., vol. 17, no. 4, pp. 523-543, 1989.
[22] R. B. Martin, "Optimal control drug scheduling of cancer chemotherapy," Automatica, vol. 28, no. 6, pp. 1113-1123, Nov. 1992

[23] R. Martin and K. L. Teo, Optimal Control of Drug Administration in Cancer Chemotherapy. Singapore: World Scientific, 1994, ch. 9, pp. 112-141.

[24] Z. Michalewicz, J. B. Krawczyk, M. Kazemi, and C. Z. Janikow, "Genetic algorithms and optimal control problems," in Proc. IEEE Conf. Decision and Control, Dec. 1990, vol. 3, pp. 1664-1666.

[25] Y. S. Ong and A. J. Keane, "Meta-Lamarckian learning in memetic algorithms," IEEE Trans. Evol. Comput., vol. 8, no. 2, pp. 99-110, Apr. 2004.

[26] Y. S. Ong, M. H. Lim, N. Zhu, and K. W. Wong, "Classification of adaptive memetic algorithms: A comparative study," IEEE Trans. Syst., Man Cybern. B, Cybern., vol. 36, no. 1, pp. 141-152, Feb. 2006.

[27] J. C. Panetta and J. Adam, "A mathematical model of cycle-specific chemotherapy," Math. Comput. Model., vol. 22, no. 2, pp. 67-82, Jul. 1995.

[28] A. Rundell, R. DeCarlos, V. Balakrishnan, and H. HogenEsch, "Systematic method for determining intravenous drug treatment strategies aiding the humoral immune response," IEEE Trans. Biomed. Eng., vol. 45, no. 4, pp. 429-439, Apr. 1998.

[29] M. Villasana and G. Ochoa, "Heuristic design of cancer chemotherapies," IEEE Trans. Evol. Comput., vol. 8, no. 6, pp. 513-521, Dec. 2004.

[30] J. J. Westman, B. R. Fabijonas, D. L. Kern, and F. B. Hanson, "Cancer treatment using multiple chemotheraputic agents subject to drug resistance," in Proc. 15th Int. Symp. Mathematical Theory Networks and Syst., paper 27126, 2002.

[31] _ - "Compartmental model for cancer evolution: Chemotherapy and drug resistance," Math. Biosci., 2001, submitted for publication. 